Research Paper

\title{
Systemic Inflammation Biomarkers Predict Survival in Patients of Early Stage Non-Small Cell Lung Cancer Treated With Stereotactic Ablative Radiotherapy - A Single Center Experience
}

\author{
Hui Luo ${ }^{1}$, Hong Ge ${ }^{2 凶}$, Yingying Cuiं ${ }^{2}$ Jiangong Zhang 3 , Ruitai Fan ${ }^{4}$, Anping Zheng 5 , Xiaoli Zheng², Yanan \\ Sun ${ }^{2}$ \\ 1. Division of Graduate, The Second Clinical Medical School and the Second Affiliated Hospital of Zhengzhou University, Zhengzhou, China; \\ 2. Department of Radiation Oncology, The Affiliated Cancer Hospital of Zhengzhou University, Zhengzhou, China; \\ 3. Division of Scientific Research and Education, The Affiliated Cancer Hospital of Zhengzhou university, Zhengzhou, China; \\ 4. Department of Radiation Oncology, The First Affiliated Hospital of Zhengzhou University, Zhengzhou, China; \\ 5. Department of Radiation Oncology, Anyang Cancer Hospital, Anyang, China. \\ $\triangle$ Corresponding author: Hong Ge, Department of Radiation Oncology, The affiliated Cancer Hospital of Zhengzhou University, No. 127 Dongming Road, \\ Zhengzhou, 450008, Henan province, China, Tel.: [86-0371-65587765], Fax: [86-0371-65587713], E-mail: progh@gs.zzu.edu.cn or gehong616@126.com \\ ( $)$ Ivyspring International Publisher. This is an open access article distributed under the terms of the Creative Commons Attribution (CC BY-NC) license \\ (https://creativecommons.org/licenses/by-nc/4.0/). See http://ivyspring.com/terms for full terms and conditions.
}

Received: 2017.06.30; Accepted: 2017.10.24; Published: 2018.01.01

\begin{abstract}
Background: Increasing evidence indicates a relationship between systemic inflammation and survival following treatment in various tumors. However, the correlation of systematic inflammation with survival after stereotactic ablative radiotherapy (SABR) in early stage non-small cell lung cancer (NSCLC) has not been well established.

Patients and methods: We retrospectively analyzed patients with newly diagnosed early stage NSCLC treated with SABR in a single institution from 2011 to 2015. The neutrophil-lymphocyte ratio (NLR), platelet-lymphocyte ratio (PLR), and lymphocyte- monocyte ratio (LMR) were calculated as systemic inflammation biomarkers. Overall survival (OS) was the first end-point. Receiver operating characteristic (ROC) was used to determine cut-off points for OS. Univariate and multivariate Cox proportional hazards regression were used to investigate the potential factors associated with OS.

Results: In the 63 patients who were eligible for analysis. The median follow up after SBRT was 29.5 months (range 8-67 months) while the 3-year OS was 74.2\%. Based on ROC analysis, optimal cut-off values of NLR, PLR, and LMR were 2.06, 199.55 and 4.0, respectively. Significant survival benefit was found in the NLR $\leq 2.06$ group $(p=0.028)$, PLR $\leq 199.55$ group $(p=0.001)$, and LMR>4.0 group $(p=0.046)$. Univariate analysis indicated that low NLR $(p=0.011)$, low PLR $(p=0.003)$, and high LMR $(p=0.014)$ were correlated with improved survival. Multivariate analysis indicated that high PLR $(p=0.033)$ and low LMR $(p=0.046)$ were independent prognostic factors for poor survival.

Conclusions: In patients of early stage NSCLC who received SABR, pretreatment NLR, PLR, and LMR could be considered useful prognostic indicators of OS. These metrics may provide reliable and convenient predictors to identify patients who would benefit from SABR.
\end{abstract}

Key words: non-small cell lung cancer; neutrophil-lymphocyte ratio; platelet-lymphocyte ratio; lymphocytemonocyte ratio; stereotactic ablative radiotherapy

\section{Introduction}

Lung cancer has become the most frequent cause of cancer death across the world, with NSCLC representing the most prevalent type [1,2]. In the past few decades, much progress has been made in identifying and treating lung cancer. Though the 5 -year OS for patients with locally advanced and 
advanced stage NSCLC remains poor, patients with early stage (stage IA, IB or II) have the largest possibility of cure with advances in surgery resection and radiation therapy [3-5]. SABR, also called stereotactic body radiotherapy (SBRT) is an alternative therapy that is widely used in the management of early stage NSCLC. SABR has shown some success in treating patients who were either medically inoperable or refuse surgery [6]. For patients who were operable, SABR has also been investigated and demonstrated promising results [7]. As SABR becomes increasingly used, it is vitally important to select eligible patients that may benefit from this treatment protocol.

Systemic inflammation is the result of pro-inflammatory cytokine secretion by immune-related cells after the activation of immune system. Biomarkers indicative of inflammation, such as white cell counts and acute phase proteins, have been repeatedly demonstrated to have prognostic value $[8,9]$. Over the last few decades, systemic inflammation has been revealed as both an etiologic factor and physiological response advanced cancer [10]. Recent findings have identified that cancer associated inflammation plays a critical role in the progression of different malignancies. Prognostic markers of systemic inflammation, including neutrophil-lymphocyte ratio (NLR), platelet-lymphocyte ratio (PLR), and lymphocytemonocyte ratio (LMR) are simple to derive, inexpensive, and have been evaluated and found to correlate with disease outcomes in multiple tumors $[11,12]$. However, most of these studies focus primarily on surgery, chemotherapy and conventional radiotherapy, with very little emphasis placed on early stage NSCLC patients who received SABR.

The cost and relative ease of deriving NLR, PLR, and LMR measurements from complete blood count (CBC) make them attractive biomarker candidates. However, it is unknown whether these biomarker candidates have prognostic value for patients that have received SABR in early stage NSCLC. Thus, we investigated the relationship between systemic inflammation biomarkers and survival of early stage NSCLC patients treated with SABR.

\section{Material and Methods}

\section{Patient population}

This study was approved by the institutional review board of the Affiliated Cancer Hospital of Zhengzhou University. Clinical and treatment data of consecutive NSCLC patients who underwent SABR at our institution from 2011 to 2015 were retrospectively analyzed. The main inclusion criteria were: early stage diagnosis (T1-2N0M0), age $\geq 18$ years, availability of CBC results prior to SABR, and no prior history of malignant tumors. Patients with metastatic lung cancer, small cell lung cancer, local-regional recurrence disease, or those receiving SABR with palliative intent were excluded. The reasons for the included patients who received SABR were: elderly, medically inoperable, refuse surgery.

\section{Management}

Prior to treatment, a computed tomography (CT) imaging of the thorax, magnetic resonance imaging of the brain, as well as blood samples were obtained. Pathologic diagnosis was conducted after voluntary biopsies. The $7^{\text {th }}$ edition of the TNM classification for lung cancer by the IASLC (International Association for the Study of Lung Cancer) was used for tumor staging [13]. A written informed consent was obtained from each patient before treatment.

For treatment planning, all patients were immobilized with arms above head within a custom stereotactic frame in the supine position. A stimulation scan was conducted with a 16-slice CT scan for all patients (Brilliance CT, Big Bore, Philips Healthcare, Andover, MA), both 3-dimensional CT (3DCT) and 4-dimensional CT images (4DCT) were acquired. Moreover, 4DCT image datasets for 10 phase bins of the respiratory cycle were generated during free breathing [14]. The maximum intensity projection (MIP) dataset was calculated based on assigning the highest density value for each pixel throughout the 10 phases of 4 DCT images [15]. Tumor volume delineation has been reported in our previous studies $[16,17]$. The gross target volume (GTV) was delineated on the 3DCT images, the internal target volume (ITV) was contoured on the MIP datasets, and combined ITV was generated by combining of GTV and ITV. The planning target volume (PTV) was defined as the combined ITV plus a $5-\mathrm{mm}$ isotropic expansion. Dosimetry required that the prescription dose cover $95 \%$ of the PTV. Both Exac Trac and cone-beam CT were performed to confirm the position of the target during daily setup. Volumetric modulated arc therapy technique was adopted (TureBeam SN1403 accelerator, Varian Medical Systems).

The SABR regimens were designed according to size and location of the tumors. The most used treatment protocols were: $48 \mathrm{~Gy}$ in 4 fractions for tumors with a maximum diameter less than $3 \mathrm{~cm}, 50$ Gy in 5 factions and 55 Gy in 5 fractions for tumors larger than $3 \mathrm{~cm}$ in size. Patient follow up, including a clinical examination and computed tomography imaging, was typically scheduled every 3 months for 
the first 2 years after the completion of treatment and every 6 months thereafter.

\section{Statistical analysis}

The following laboratory parameters were collected from the CBC 1-3 days before SABR: neutrophil count, lymphocyte count, platelet count, and monocyte count. NLR was calculated with dividing the neutrophil count by the lymphocyte count. PLR was calculated as the platelet count divided by the lymphocyte count; similarly, LMR was calculated by dividing the lymphocyte count to the monocyte count. OS was the primary endpoint and defined from the date of SABR to date of death, the last follow up. Survival analyses were evaluated via the Kaplan-Meier methodology; the log-rank test was used to detect potential differences. Receiver operating characteristic (ROC) analysis was applied to determine the optimal cut-off values of NLR, PLR and LMR for survival. These calculated values were used for subsequent Kaplan-Meier analysis. In order to assess different SABR fraction regimes on survival, we calculated the BED10 (biologically effective dose) according to the linear-quadratic equation. To identify potential prognostic factors, univariate and multivariate Cox proportional hazard regression

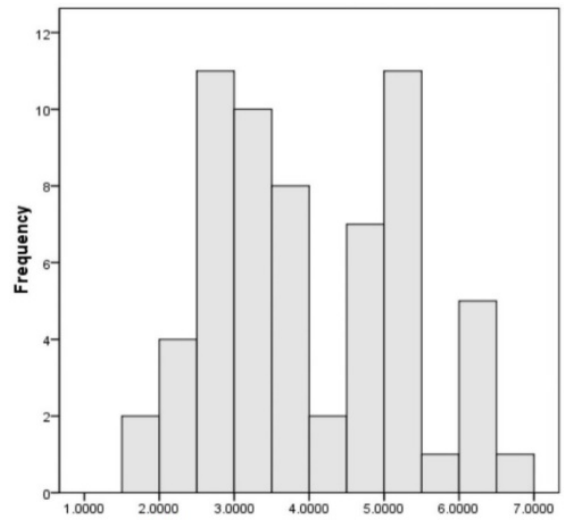

(A) Neutrophil Count $(\times 10 \%)$

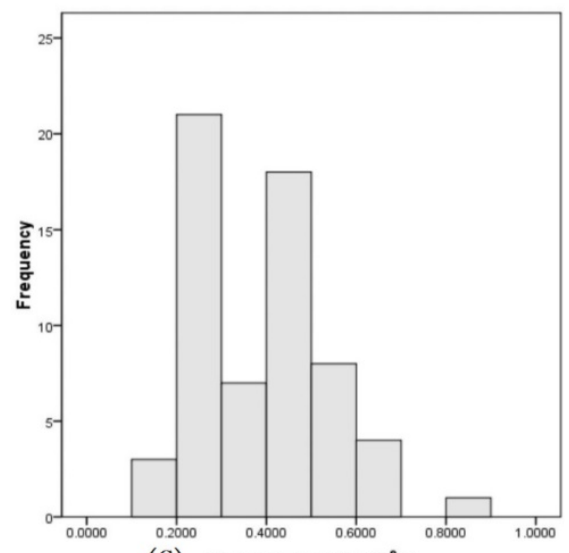

(C) Monocyte Count (x10\%)
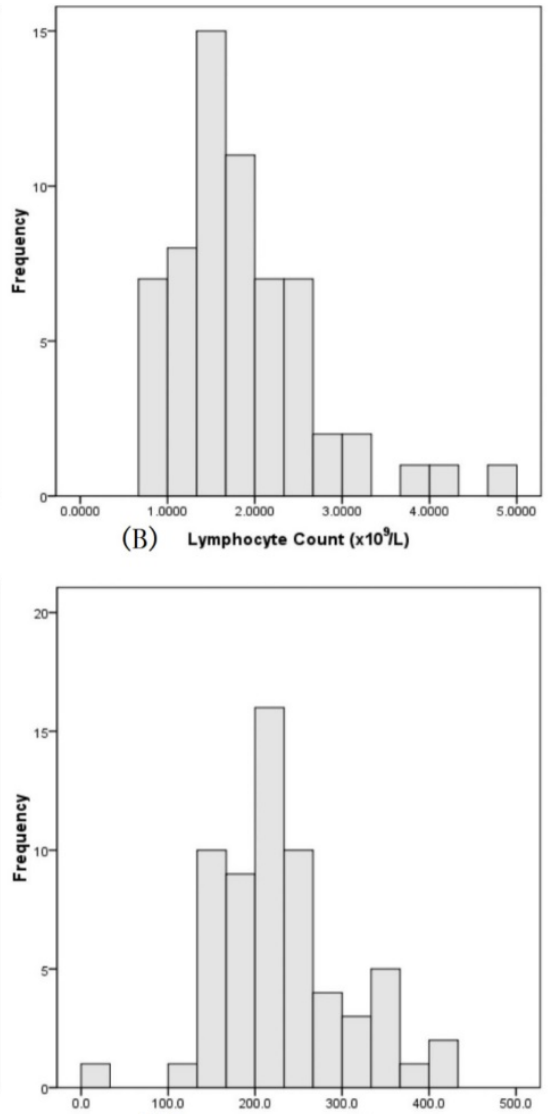

(D) Platelet Count ( $\times 10^{9} \mathrm{~L}$ )
(B) Lymphocyte Count $\left(\times 10^{9}\right.$ L)

Figure 1. Pretreatment distribution of neutrophils, lymphocytes, monocytes, and platelets. models were analyzed. Spearman's-rho analysis was utilized to determine the correlation between systemic inflammation biomarkers and clinic-pathological features. Statistical analyses were calculated using the statistical package for social sciences (SPSS) version 20.0 (IBM Software Group, Chicago, USA). All statistical tests were two-sided, and the level of significance was set to $5 \%(p<0.05)$ for all of the statistical analyses.

\section{Results}

\section{Patient characteristics}

There were 216 patients who treated with SABR in our institution. Under careful review, 63 patients met the inclusion criteria and were eligible for analysis. Median follow up time was 29.5 months (range, 8-67 months). Median age of the whole cohort was 73 years (range, $44-89$ years). Most of the selected patients were men, the proportion of male individuals was about $77.8 \%$ whereas only $22.2 \%$ female patients were included. Pretreatment biopsy for histological diagnosis was available for $74.6 \%$ of individuals; the remaining patients refused to provide biopsies. The main characteristics of the selected patients are illustrated in Table 1.

\section{Laboratory values}

Laboratory values of all included patients were available. The median neutrophil count was $3.96 \times 10^{9} \quad$ cells $/ \mathrm{L}$ (range: 1.80-6.87×109 cells/L). The median lymphocyte count was $1.85 \times 10^{9}$ cells/L (range: $0.68-4.77 \times 10^{9}$ cells/L). The median platelet count was $228.53 \times 10^{9}$ cells/L (range: 115.0-421.0 $\times 10^{9}$ cells/L). The median monocyte count was $0.38 \times 10^{9}$ cells/L (range: $0.13-0.80$ $\times 10^{9}$ cells/L). Details are illustrated in Figure 1. Median NLR was 2.47 (range: 0.86-7.29), median PLR was 140.37 (range: 4.75-315.71), and median LMR was 5.25 (range: 1.33-11.14).

\section{Survival analyses}

ROC curves were calculated and the optimal cut-off values for NLR, PLR, and LMR were 2.06, 199.55, and 4.0, respectively. The 3 -year OS of the entire cohort was $74.2 \%$. Based on the cut-off points, patients were divided into two groups (low value group and high 
value group). A NLR $\leq 2.06$, a PLR $\leq 199.55$, and a LMR $>4.0$ optimally differentiated OS. We analyzed the two groups by Kaplan-Meier survival analysis, a high NLR ( $p=0.028$; Figure 2a), a high PLR $(p=0.001$; Figure $2 \mathbf{b})$ as well as a low LMR ( $p=0.046$; Figure $2 c)$ were associated with significantly decreased OS.

Table 1. Patients and disease characteristics.

\begin{tabular}{ll}
\hline Variable & Frequency \\
\hline Patients (n) & 63 \\
Age (years) & $72.72 \pm 9.01$ \\
$\quad$ Mean & 73.00 \\
Median & $44-89$ \\
Range & \\
Sex (n) & $14(22.2 \%)$ \\
$\quad$ Women & $49(77.8 \%)$ \\
$\quad$ Men & \\
Smoke status & $10(15.9 \%)$ \\
$\quad$ Ex-smoker & $32(50.8 \%)$ \\
Current smoker & $21(33.3 \%)$ \\
$\quad$ Non-smoker & \\
Histology (n) & $25(39.7 \%)$ \\
$\quad$ Squamous cell carcinoma & $22(34.9 \%)$ \\
Adenocarcinoma & $16(25.4 \%)$ \\
Unknown & \\
Tumor stage (n) & $44(69.8 \%)$ \\
T1 & $19(30.2 \%)$ \\
T2 & \\
Radiotherapy (n) & $14(22.2 \%)$ \\
48 Gy in 4 factions & $25(39.7 \%)$ \\
50 Gy in 5 factions & $8(12.7 \%)$ \\
55 Gy in 5 factions & $16(25.4 \%)$ \\
Other factions &
\end{tabular}

Based on univariate analysis for OS, decreased NLR $(\mathrm{P}=0.011$, hazard ratio $[\mathrm{HR}]=1.489[95 \%$ confidence interval (CI), 1.096-2.021]), elevated LMR $(\mathrm{P}=0.014, \mathrm{HR}=0.601$ [95\% CI, 0.402-0.900]), and decreased PLR $(\mathrm{P}=0.003, \mathrm{HR}=1.012[95 \% \mathrm{CI}$, 1.004-1.019]) correlated with better OS (Table 2).

In order to determine the independent prognostic factors for OS, we also performed multivariate analyses by using Cox proportional hazard models. Decreased LMR $(\mathrm{P}=0.046, \mathrm{HR}=0.544$ [95\% CI, 0.299-0.989]), and elevated PLR ( $\mathrm{P}=0.033$, HR $=1.018[95 \% \mathrm{CI}, 1.001-1.034])$ were correlated with poor OS (Table 2).

\section{Correlations between Systemic inflammation biomarkers and clinic-pathological features}

As illustrated in Table 3, the correlations between Systemic inflammation biomarkers and clinic-pathological features were also calculated using Spearman's-rho analysis. The following correlations achieved statistical significance $(p<0.05)$ : NLR and gene mutation, NLR and smoke status, PLR and tumor stage, LMR and gene mutation, as well as LMR and smoke status. However, their correlation coefficients were too low, which suggests weak correlations.

\section{Discussion}

The present study demonstrates that Systemic inflammation biomarkers, as measured by NLR, PLR, and LMR, are correlated with survival in patients of early stage NSCLC who received SABR. Our results suggest that an elevated LMR (> 4.0) and a decreased PLR ( $\leq$ 199.55), were indicative of favorable independent prognostic factors for this patient population. In addition, our analysis also suggests that NLR, PLR, and LMR are weakly correlated with other clinical-pathologic features of early stage NSCLC patients who received SABR.

A century ago, the bilateral influence of inflammation and cancer was noticed by Rudolf Virchow [18]. In previous studies, the prognostic potential of systemic inflammation biomarkers was assessed in hematological cancers and various types of solid tumors. Hu et al conducted a retrospective study detailing a positive correlation between LMR and survival in operable NSCLC; the result indicated LMR was an effective prognostic factor [19]. The NLR has shown potential for use as a prognostic biomarker in patients of early stage NSCLC undergoing resection [20]. A systematic review by Guthrie has examined the clinical utility of the NLR and its association with patient outcomes in different kind of tumors; they found that the NLR could serve as an independent prognostic factor in patients undergoing surgery, neoadjuvant treatment followed by surgery, definitive chemoradiotherapy, and groups with inoperable tumors [21]. Lan et al recently reported the PLR as a good prognostic factor for OS in NSCLC patients undergoing radical lung cancer surgery [22]. However, the use of systemic inflammation biomarkers are not well characterized in patients with early stage NSCLC treated with SABR.

A single institution study by Shaverdian found an elevated pretreatment NLR and PLR independently predicted poor OS [23]. Likewise, a retrospective study by Cannon also indicated elevated NLR and PLR were associated with poor survival [24]. Giuliani et al demonstrated that NLR and LMR were independently correlated with $O S$ in early stage NSCLC patients who were treated with SABR [25]. In the present work, we combined the NLR, PLR, and LMR metrics in order to analyze their collective contribution to OS. We calculated the cut-off value of NLR, PLR, and LMR to predict survival; the final outcomes were similar to Shaverdian's research insofar that our study suggests that an elevated pretreatment LMR, in conjunction with a decreased PLR and NLR were associated with better OS. 


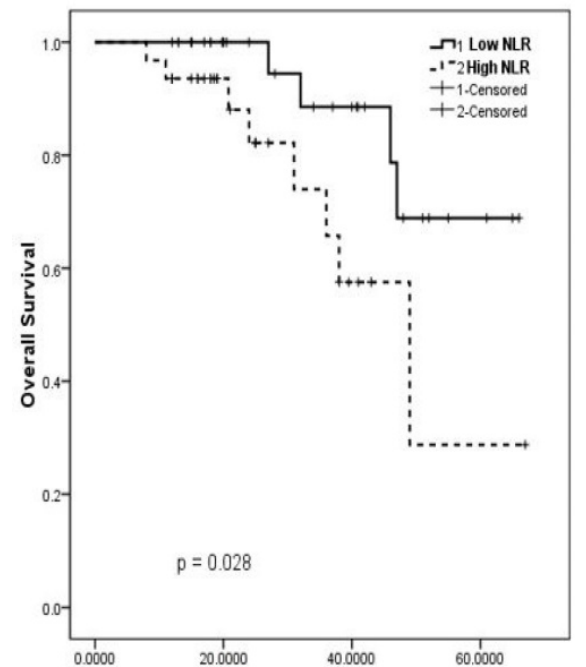

(A) Time (months)

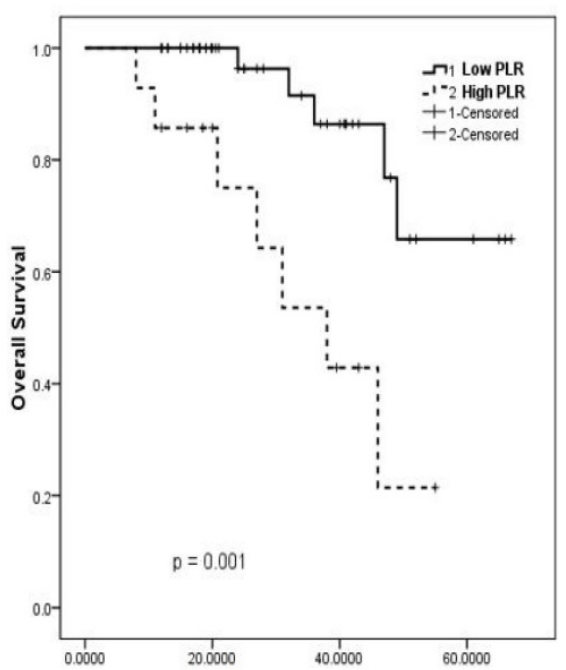

(B) Time (months)

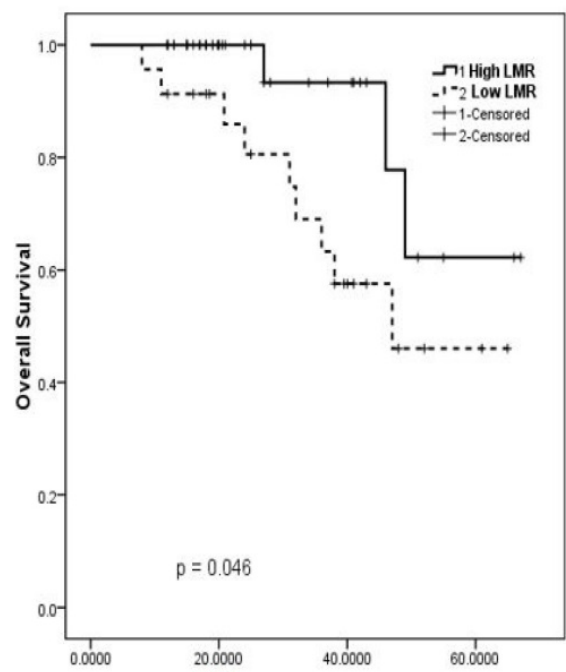

(C) Time (months)

Figure 2. Overall survival in early stage patients. A: Survival based on neutrophil to lymphocyte ratio. Solid line-NLR $\leq 2.06$, dashed line-NLR $>2.06$. B: Survival based on platelet to lymphocyte ratio. Solid blue-PLR $\leq 199.5$, dashed green-PLR $>199.5$. C: Survival based on lymphocyte to monocyte ratio. Solid blue-PLR $>4.0$, dashed green-PLR 4 4.0. Abbreviations: NLR: neutrophil-lymphocyte ratio; PLR: platelet-lymphocyte ratio; LMR: lymphocyte- monocyte ratio.

Table 2. Cox proportional hazards regression for OS.

\begin{tabular}{|c|c|c|c|c|}
\hline \multirow[t]{2}{*}{ Variable } & \multicolumn{2}{|l|}{ Univariate analysis } & \multicolumn{2}{|l|}{$\begin{array}{l}\text { Multivariate } \\
\text { analysis }\end{array}$} \\
\hline & HR $(95 \% \mathrm{CI})$ & $\mathrm{P}$ & HR (95\%CI) & $\mathrm{P}$ \\
\hline Age $(>65$ vs $\leq 65)$ & $1.047(0.975-1.125)$ & 0.203 & & \\
\hline Sex (male vs female) & $1.108(0.299-4.100)$ & 0.878 & & \\
\hline Smoke status (yes vs no) & $0.557(0.164-1.895)$ & 0.349 & & \\
\hline Tumor stage (T1 vs $\mathrm{T} 2$ ) & $2.402(0.752-7.675)$ & 0.139 & & \\
\hline BED ( >106Gy vs $\leq 106$ Gy) & $1.032(0.958-1.113)$ & 0.405 & & \\
\hline NLR $(>2.06$ vs $\leq 2.06)$ & $1.489(1.096-2.021)$ & 0.011 & & \\
\hline PLR ( >199.55 vs $\leq 199.55)$ & $1.012(1.004-1.019)$ & 0.003 & $\begin{array}{l}1.018 \\
(1.001-1.034)\end{array}$ & 0.033 \\
\hline $\operatorname{LMR}(>4.0$ vs $\leq 4.0)$ & $0.601(0.402-0.900)$ & 0.014 & $\begin{array}{l}0.544 \\
(0.299-0.989)\end{array}$ & 0.046 \\
\hline
\end{tabular}

Abbreviations: NLR, neutrophil-to-lymphocyte ratio; PLR, platelet-to-lymphocyte ratio; LMR, lymphocyte- monocyte ratio. CI, confidence interval; HR, hazard ratio; BED, biological effective dose; OS, overall survival.

Table 3. Correlations between pretreatment immune parameters and clinic- pathological features.

\begin{tabular}{lllllll}
\hline \multirow{2}{*}{$\begin{array}{l}\text { Clinic-pathological } \\
\text { features }\end{array}$} & \begin{tabular}{l} 
NLR \\
\cline { 2 - 6 }
\end{tabular} & $\begin{array}{l}\text { Correlation } \mathrm{P} \\
\text { coefficient }\end{array}$ & \multicolumn{2}{l}{$\begin{array}{l}\text { PLR } \\
\text { Correlation P } \\
\text { coefficient }\end{array}$} & $\begin{array}{l}\text { LMR } \\
\text { Correlation P } \\
\text { coefficient }\end{array}$ \\
\hline Age & -0.019 & 0.880 & 0.049 & 0.700 & -0.192 & 0.132 \\
Gene mutation & -0.262 & 0.038 & -0.195 & 0.125 & -0.339 & 0.007 \\
Smoke status & -0.317 & 0.011 & 0.094 & 0.466 & -0.360 & 0.004 \\
Tumor stage & -0.107 & 0.406 & 0.289 & 0.022 & -0.219 & 0.085 \\
BED & -0.007 & 0.955 & 0.031 & 0.808 & 0.022 & 0.866 \\
\hline
\end{tabular}

Abbreviations: NLR, neutrophil-to-lymphocyte ratio; PLR, platelet-to-lymphocyte ratio; LMR, lymphocyte- monocyte ratio. BED, biological effective dose.

The immune system plays an important role in resisting or eradicating tumor formation and progression. Chronic inflammation induces several molecular cascades in cancer cells that facilitate immune cell evasion and tumor invasion [26]. Indeed, cancer incidence is increased when there are deficiencies in the development or function of CD8+ cytotoxic T lymphocytes and natural killer cells [27]. An elevated NLR, PLR, and a decreased LMR indicate systemic inflammation, which can lead to increased resting energy expenditure, hypoalbuminemia and malnutrition, eventually resulting in weight loss and tumor progression thereby leading to increased mortality [28, 29]. Other biomarkers such as transforming growth factor beta (TGF- $\beta$ ) are also involved in inflammation in cancer. Previous studies demonstrated that tumor cells could secreting TGF- $\beta$ to inhibit the function of CD8+ cytotoxic $\mathrm{T}$ lymphocytes and natural killer cells [30].

Necroptosis is involved in the regulation of cancer. Radiotherapy can promote necroptosis of tumor cells. High dose radiation, such as SABR, could promote necroptosis through the caspase-3 signal pathway. Necroptotic cancer cells are capable of providing specific antigens and inflammatory stimuli for dendritic cells, while neutrophils, monocytes, and macrophages could produce pro-inflammatory cytokines and chemokines. Cytokine and chemokine production subsequently enhances antigenpresentation and $\mathrm{T}$ lymphocyte activation, thereby inducing anti-cancer immunity [31].

Mesenchymal stem cells (MSCs) are multipotent stromal cells that can differentiate into a variety of cell types. Chronic inflammation of the stroma could result in impairment of the immune system through an MSC dependent mechanism [32]. SABR could enhance MSC migration to the tumor microenvironment and generate pericytes, which 
have been associated with local tumor recurrence, drug resistance, and passive immune evasion [33, 34].

Several researchers have shown the success of SABR as an effective, noninvasive treatment for patients with medically inoperable early stage NSCLC [35-38]. Findings from a retrospective study showed that the OS of SABR-treated NSCLC patients was 77\% at 3 years [23]. Similarly, the 3-year OS reported by the Japanese Clinical Oncology Group 0403 trial was $76 \%$ [39]. In a propensity score-matched analysis by Verstegen, the 3-year OS of early stage NSCLC who received SABR was $79.6 \%$ [40]. The 3-year OS in the current study was similar to the above research. The combination of immunotherapy and SABR has been under investigation recently, as evidence showed synergy between immunotherapy and SABR in eliminating micrometastastatic disease [41, 42]. The NLR, PLR, and LMR metrics that are derived from $\mathrm{CBC}$ could be helpful in selecting the subset of early stage NSCLC patients who may benefit from SABR and its combination with immunotherapy.

There were some inherent limitations in our study. The present research was performed in a single medical center and only a small number of patients were included for analysis. The radiation doses received for the whole patient population were relatively uniform. Some patients also possessed additional medical co-morbidities such as coronary artery disease, which may influence systemic inflammation biomarkers and overall survival. Hence our findings should be interpreted with caution and need confirmation in larger prospective researches.

\section{Conclusions}

In conclusion, our data suggest that systemic inflammation biomarkers, including NLR, PLR, and LMR were correlated with OS in early stage NSCLC patients treated with SABR. The PLR and LMR could be regarded as useful independent prognostic factors in daily clinical practice. Future clinical trials based on larger populations are needed to determine the optimal cut-off values of NLR, PLR, and LMR.

\section{Abbreviations}

BED: biological effective dose; CBC: complete blood count; CI: confidence interval; CT: computed tomography; GTV: gross target volume; HR: hazard ratio; IASLC: International Association for the Study of Lung Cancer; ITV: internal target volume; LMR: lymphocyte- monocyte ratio; MIP: maximum intensity projection; MSC: Mesenchymal stem cell; NLR: neutrophil-lymphocyte ratio; NSCLC: non-small cell lung cancer; OS: overall survival; PLR: platelet-lymphocyte ratio; PTV: planning target volume; ROC: Receiver operating characteristic;
SABR: stereotactic ablative radiotherapy; TGF- $\beta$ : transforming growth factor beta.

\section{Acknowledgements}

We appreciate Kyle Vaughn Laster (Hormel Cancer Institute, USA) for editing the language of the present manuscript.

\section{Grant support}

This work was supported by the National Natural Science Foundation of China [grant no. 81372436, 81773230], the Science and Technology Innovative Research Group of Zhengzhou city [grant no. 121PCXTD524], and the Joint Research Funds of Henan province and the Minister of Health of China [grant no. 201201009].

\section{Competing Interests}

The authors have declared that no competing interest exists.

\section{References}

1. Torre LA, Siegel RL, Jemal A. Lung Cancer Statistics. Advances in experimental medicine and biology. 2016; 893: 1-19.

2. Siegel RL, Miller KD, Jemal A. Cancer Statistics, 2017. CA: a cancer journal for clinicians. 2017; 67: 7-30.

3. Luo H, Yu X, Liang N, et al. The effect of induction chemotherapy in patients with locally advanced nonsmall cell lung cancer who received chemoradiotherapy: A systematic review and meta-analysis. Medicine. 2017; 96: e6165.

4. Howington JA, Blum MG, Chang AC, Balekian AA, Murthy SC. Treatment of stage I and II non-small cell lung cancer: Diagnosis and management of lung cancer, 3rd ed: American College of Chest Physicians evidence-based clinical practice guidelines. Chest. 2013; 143: e278S-313S.

5. Rosenzweig KE, Chang JY, Chetty IJ, et al. ACR appropriateness criteria nonsurgical treatment for non-small-cell lung cancer: poor performance status or palliative intent. Journal of the American College of Radiology : JACR. 2013; 10: 654-64.

6. Sun B, Brooks ED, Komaki RU, et al. 7-year follow-up after stereotactic ablative radiotherapy for patients with stage I non-small cell lung cancer: Results of a phase 2 clinical trial. Cancer. 2017.

7. Chang JY, Senan S, Paul MA, et al. Stereotactic ablative radiotherapy versus lobectomy for operable stage I non-small-cell lung cancer: a pooled analysis of two randomised trials. The Lancet Oncology. 2015; 16: 630-7.

8. McMillan DC. Cancer and systemic inflammation: stage the tumour and stage the host. British journal of cancer. 2013; 109: 529.

9. Gabay C, Kushner I. Acute-phase proteins and other systemic responses to inflammation. The New England journal of medicine. 1999; 340: 448-54.

10. Martin HL, Ohara K, Kiberu A, Van Hagen T, Davidson A, Khattak MA. Prognostic value of systemic inflammation-based markers in advanced pancreatic cancer. Internal medicine journal. 2014; 44: 676-82.

11. Proctor MJ, McMillan DC, Morrison DS, Fletcher CD, Horgan PG, Clarke SJ. A derived neutrophil to lymphocyte ratio predicts survival in patients with cancer. British journal of cancer. 2012; 107: 695-9.

12. Templeton AJ, McNamara MG, Seruga B, et al. Prognostic role of neutrophil-to-lymphocyte ratio in solid tumors: a systematic review and meta-analysis. Journal of the National Cancer Institute. 2014; 106: dju124.

13. Goldstraw P, Crowley J, Chansky K, et al. The IASLC Lung Cancer Staging Project: proposals for the revision of the TNM stage groupings in the forthcoming (seventh) edition of the TNM Classification of malignant tumours. Journal of thoracic oncology : official publication of the International Association for the Study of Lung Cancer. 2007; 2: 706-14.

14. Underberg RW, Lagerwaard FJ, Slotman BJ, Cuijpers JP, Senan S. Benefit of respiration-gated stereotactic radiotherapy for stage I lung cancer: an analysis of $4 \mathrm{DCT}$ datasets. International journal of radiation oncology, biology, physics. 2005; 62: 554-60.

15. Bradley JD, Nofal AN, El Naqa IM, et al. Comparison of helical, maximum intensity projection (MIP), and averaged intensity (AI) 4D CT imaging for stereotactic body radiation therapy (SBRT) planning in lung cancer. Radiotherapy and oncology : journal of the European Society for Therapeutic Radiology and Oncology. 2006; 81: 264-8.

16. Ge H, Cai J, Kelsey CR, Yin FF. Quantification and minimization of uncertainties of internal target volume for stereotactic body radiation therapy 
of lung cancer. International journal of radiation oncology, biology, physics. 2013; 85: 438-43.

17. Sun $\mathrm{Y}, \mathrm{Ge} \mathrm{H}, \mathrm{Cheng} \mathrm{S}$, et al. Evaluation of interfractional variation of the centroid position and volume of internal target volume during stereotactic body radiotherapy of lung cancer using cone-beam computed tomography. Journal of applied clinical medical physics. 2016; 17: 5835.

18. Balkwill F, Mantovani A. Inflammation and cancer: back to Virchow? Lancet. 2001; 357: 539-45.

19. Hu P, Shen H, Wang G, Zhang P, Liu Q, Du J. Prognostic significance of systemic inflammation-based lymphocyte- monocyte ratio in patients with lung cancer: based on a large cohort study. PloS one. 2014; 9: e108062.

20. Sarraf KM, Belcher E, Raevsky E, Nicholson AG, Goldstraw P, Lim E. Neutrophil/lymphocyte ratio and its association with survival after complete resection in non-small cell lung cancer. The Journal of thoracic and cardiovascular surgery. 2009; 137: 425-8.

21. Guthrie GJ, Charles KA, Roxburgh CS, Horgan PG, McMillan DC, Clarke SJ. The systemic inflammation-based neutrophil-lymphocyte ratio: experience in patients with cancer. Critical reviews in oncology/hematology. 2013; 88: 218-30.

22. Lan $\mathrm{H}$, Zhou L, Chi D, et al. Preoperative platelet to lymphocyte and neutrophil to lymphocyte ratios are independent prognostic factors for patients undergoing lung cancer radical surgery: A single institutional cohort study. Oncotarget. 2017; 8: 35301-10.

23. Shaverdian N, Veruttipong D, Wang J, Schaue D, Kupelian P, Lee P. Pretreatment Immune Parameters Predict for Overall Survival and Toxicity in Early-Stage Non-Small-Cell Lung Cancer Patients Treated With Stereotactic Body Radiation Therapy. Clinical lung cancer. 2016; 17: 39-46.

24. Cannon NA, Meyer J, Iyengar P, et al. Neutrophil-lymphocyte and platelet-lymphocyte ratios as prognostic factors after stereotactic radiation therapy for early-stage non-small-cell lung cancer. Journal of thoracic oncology : official publication of the International Association for the Study of Lung Cancer. 2015; 10: 280-5.

25. Giuliani M, Sampson LR, Wong O, et al. Prognostic value of pretreatment circulating neutrophils, monocytes, and lymphocytes on outcomes in lung stereotactic body radiotherapy. Current oncology (Toronto, Ont). 2016; 23: e362-8.

26. Hanahan D, Weinberg RA. Hallmarks of cancer: the next generation. Cell. 2011; 144: 646-74.

27. Kim R, Emi M, Tanabe K. Cancer immunoediting from immune surveillance to immune escape. Immunology. 2007; 121: 1-14.

28. Falconer JS, Fearon KC, Plester CE, Ross JA, Carter DC. Cytokines, the acute-phase response, and resting energy expenditure in cachectic patients with pancreatic cancer. Annals of surgery. 1994; 219: 325-31.

29. Wang DS, Luo HY, Qiu MZ, et al. Comparison of the prognostic values of various inflammation based factors in patients with pancreatic cancer. Medical oncology (Northwood, London, England). 2012; 29: 3092-100.

30. Yang L, Pang Y, Moses HL. TGF-beta and immune cells: an important regulatory axis in the tumor microenvironment and progression. Trends in immunology. 2010; 31: 220-7.

31. Meng MB, Wang HH, Cui YL, et al. Necroptosis in tumorigenesis, activation of anti-tumor immunity, and cancer therapy. Oncotarget. 2016; 7: 57391-413.

32. Lepperdinger G. Inflammation and mesenchymal stem cell aging. Current opinion in immunology. 2011; 23: 518-24.

33. Wang HH, Cui YL, Zaorsky NG, et al. Mesenchymal stem cells generate pericytes to promote tumor recurrence via vasculogenesis after stereotactic body radiation therapy. Cancer letters. 2016; 375: 349-59.

34. Meng MB, Zaorsky NG, Deng L, et al. Pericytes: a double-edged sword in cancer therapy. Future oncology. 2015; 11: 169-79.

35. Koshy M, Malik R, Mahmood U, Husain Z, Sher DJ. Stereotactic body radiotherapy and treatment at a high volume facility is associated with improved survival in patients with inoperable stage I non-small cell lung cancer. Radiotherapy and oncology : journal of the European Society for Therapeutic Radiology and Oncology. 2015; 114: 148-54.

36. Nanda RH, Liu Y, Gillespie TW, et al. Stereotactic body radiation therapy versus no treatment for early stage non-small cell lung cancer in medically inoperable elderly patients: A National Cancer Data Base analysis. Cancer. 2015; 121: 4222-30.

37. Videtic GM, Hu C, Singh AK, et al. A Randomized Phase 2 Study Comparing 2 Stereotactic Body Radiation Therapy Schedules for Medically Inoperable Patients With Stage I Peripheral Non-Small Cell Lung Cancer: NRG Oncology RTOG 0915 (NCCTG N0927). International journal of radiation oncology, biology, physics. 2015; 93: 757-64.

38. Navarro-Martin A, Aso S, Cacicedo J, et al. Phase II Trial of SBRT for Stage I NSCLC: Survival, Local Control, and Lung Function at 36 Months. Journal of thoracic oncology : official publication of the International Association for the Study of Lung Cancer. 2016; 11: 1101-11.

39. Nagata Y, Hiraoka M, Shibata T, et al. Prospective Trial of Stereotactic Body Radiation Therapy for Both Operable and Inoperable T1N0M0 Non-Small Cell Lung Cancer: Japan Clinical Oncology Group Study JCOG0403. International journal of radiation oncology, biology, physics. 2015; 93: 989-96.

40. Verstegen NE, Oosterhuis JW, Palma DA, et al. Stage I-II non-small-cell lung cancer treated using either stereotactic ablative radiotherapy (SABR) or lobectomy by video-assisted thoracoscopic surgery (VATS): outcomes of a propensity score-matched analysis. Annals of oncology : official journal of the European Society for Medical Oncology. 2013; 24: 1543-8.
41. Filatenkov A, Baker J, Mueller AM, et al. Ablative Tumor Radiation Can Change the Tumor Immune Cell Microenvironment to Induce Durable Complete Remissions. Clinical cancer research : an official journal of the American Association for Cancer Research. 2015; 21: 3727-39.

42. Bernstein MB, Krishnan S, Hodge JW, Chang JY. Immunotherapy and stereotactic ablative radiotherapy (ISABR): a curative approach? Nature reviews Clinical oncology. 2016; 13: 516-24. 